\title{
Selection of a Remote Phosphor Structure for Phosphor- converted White LEDs with High Luminous Efficacy
}

\author{
Long Nguyen $\mathrm{Ngoc}^{1,4}$, Nguyen Doan Quoc Anh ${ }^{2, *}$, Nhu Nguyen Hong ${ }^{3,4}$, Jaroslav Zdralek ${ }^{4}$, Miroslav Voznak ${ }^{4}$ \\ ${ }^{1}$ Faculty of Applied Science, Ton Duc Thang University, Ho Chi Minh City, Vietnam \\ ${ }^{2}$ Power System Optimization Research Group, Faculty of Electrical and Electronics Engineering, Ton Duc Thang University, Ho Chi \\ Minh City, Vietnam \\ ${ }^{3}$ Saigon University of Ho Chi Minh City, Viet Nam \\ ${ }^{4}$ VSB-Technical University of Ostrava, Czech
}

\begin{abstract}
This study presents the influence of the distance between phosphor layers in the dual-layer and triple- layer remote package on luminous efficacy. During the simulation, it is recognized that an appropriate distance can produce higher luminous flux of the multi-chip white LED (WLEDs) through adjusting the distance between two and three phosphor layers. According to the research results, $0.1 \mathrm{~mm}$ is the outstanding distance between two phosphor layers so that the performance of MCW-LEDs can be accomplished the best optimal effect. In addition, the simulation results show that the dual-layer structure yielded higher optical properties than the triple - layer structure in relation to the distance.
\end{abstract}

\section{Introduction}

As a development, a next generation of remote phosphor configuration is proposed, in which the dual-layer structure is employed in the remote phosphor package to enhance luminous efficiency of pc-LEDs. A study with applying a thin silicone layer into dual-layer remote phosphor structure can yield 5\% higher lumen output than a conventional remote phosphor package at the same CCT [1-8]. Similarly, there are some approaches focusing on the phosphor structure proposed to reduce the probability of the backward light from the phosphor layer to the absorptive LED chip such as remote phosphor package with the hemispherical dome, employing a double remote micro-patterned phosphor film into pc-LEDs as well as ring remote phosphor structure [9-12]. A different propose also demonstrated that an air-gap layer embedded in the remote phosphor package offers advantages in luminous flux and chromatic stability [13- 15]. Besides, the different phosphor material arrangement has a significant role for improving the phosphor converted efficiency. A twolayer remote phosphor structure in which a SrO. $3 \mathrm{~B}_{2} \mathrm{O}_{3}: \mathrm{Sm}^{2+}$ red phosphor layer above a yellow phosphor layer can achieve more than $17 \%$ in lumen compared to the mixed red and yellow phosphor package [16-23]. However, these phosphor layers in this structure are placed adjacent to each other at a random distance with LED chip in remote phosphor package and in these studies, the specified distance between phosphor layers is not determined so it should be suggested to achieve the best optical performance.

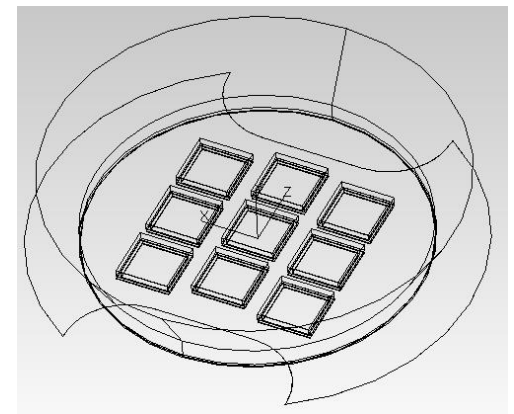

(a)

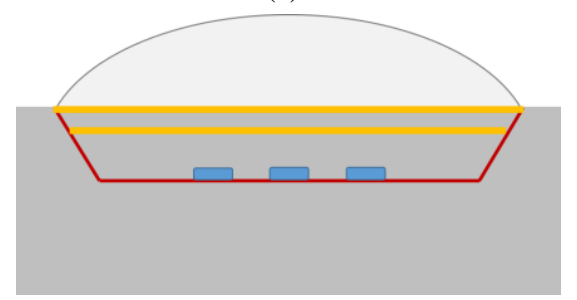

(b)

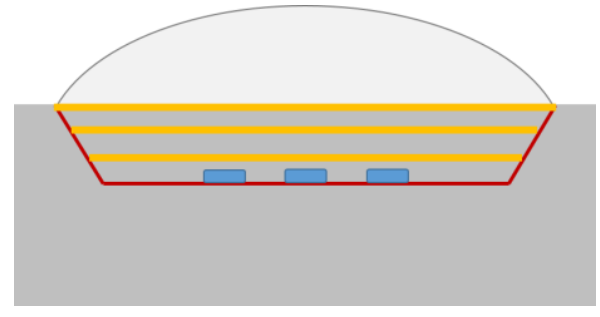

(c)

Fig. 1. (a) Simulation of the white LED package (b) Illustration of WLEDs with dual-layer (a) and triple-layer (bottom) structures.

In this research, we study the effect of the distance 
between phosphor layers and LED chip on the optical properties of both dual-layer and triple layer structures. The luminous efficiency, the color rendering ability in two and three-layer remote phosphor package versus distance between phosphors are investigated and presented. We try to look into a best position of phosphor layers within LEDs and hope to achieve an optimized design of the high efficiency LEDs. The obtained results indicate that the highest overall light output, lumen efficiency as well as better color quality of LEDs can be achieved at the distance between two phosphor layers of $0.1 \mathrm{~mm}$ for dual-layer structure and $0.6 \mathrm{~mm}$ for triple layer structure. The enhancement of efficiency was due to the improving in the utilization of blue, yellow rays within the LEDs. The simulation results show that the LED with the two-layer remote phosphor package exhibits the light extraction, color rendering ability better than triple-layer remote phosphor package.

Table 1. The actual parameters of LED chip

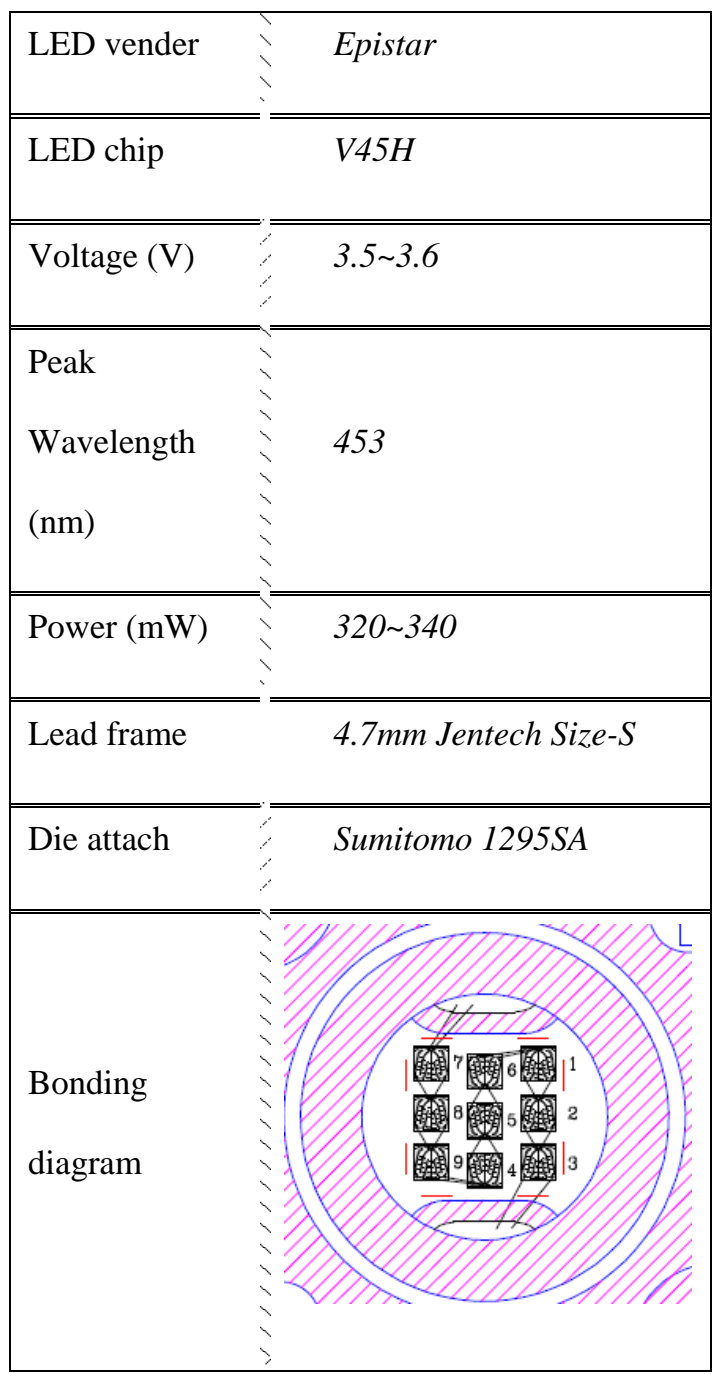

\section{Simulation}

The basic WLEDs model is made of a reflector, a phosphor layer, and a silicone layer. The reflector has a depth of $2.07 \mathrm{~mm}$, a bottom length of $8 \mathrm{~mm}$ and a surface length of $9.85 \mathrm{~mm}$. The dimensions of each blue LED chip are $1.14 \mathrm{~mm} \times 0.15 \mathrm{~mm}$. These chips are covered by the phosphor layer with a $0.08 \mathrm{~mm}$ thickness as illustrated in Fig. 1. The luminous power of each blue chip is $1.16 \mathrm{~W}$, and the light emitting wavelength is 453 $\mathrm{nm}$, see Table 1. Furthermore, the spectra values of YAG:Ce including absorption spectrum and emission spectrum are presented in Figure 2.

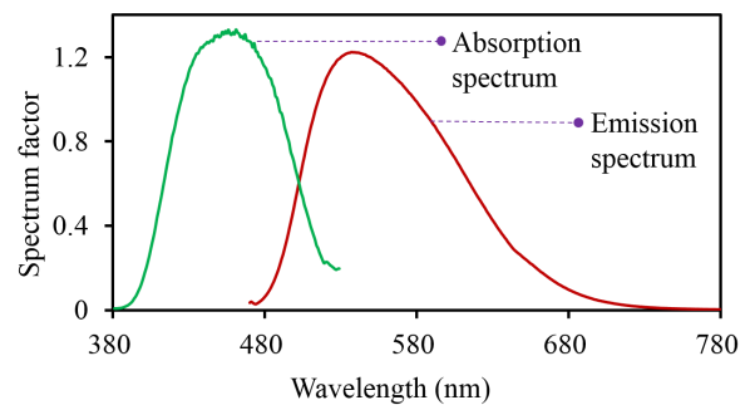

Fig. 2. Absorption spectrum and emission spectrum of YAG:Ce.

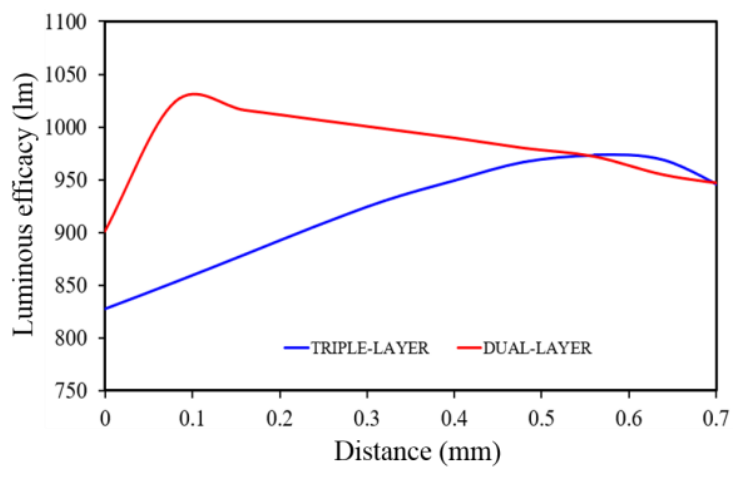

Fig. 3. Luminous efficacy of two structures.

Figure 3 depicts the influence of the distance between phosphor layers and LED chip of remote phosphor package on the lumen output. The simulation results show that the variation of the distance has a significant impact on the light extraction. For the dual-layer package, the lumen output dramatically enhances and gets the peak value with ranging from 0 to $0.1 \mathrm{~mm}$. Conversely, it tends to drop when the distance of phosphor layers continuously increases further. When the distance $d$ varies from 0 to $0.1 \mathrm{~mm}$, it means that these two phosphor layers are separated further away, the probability of light trapped in the gap between two phosphor layers will reduce. The reflected light which directly impinges on the absorptive LED chip could decrease and the transmitted light through phosphor layers can be increased, thus this improves the LED efficiency. With distance range within $0-0.1 \mathrm{~mm}$, the heat generated by the LED chip only transfers to the substrate instead of the contact surface of two phosphor layers. The increase of the distance between these phosphor layers can offer not only the high luminous flux but also produce the temperature stability of this multilayer phosphor configuration. This leads to more light be transmitted and extracted through the phosphor layers. For the distance ranging from 0.2 to $0.7 \mathrm{~mm}$, the drop of the luminous flux is attributed by the weakened 
photon extraction and the heat effect of phosphor. The blue light from LED chip will encounter the first phosphor layer and be converted to the yellow light.

However, some portion of light is lost inside the LEDs due to the backscattering, absorption and reflection, the other portion is converted to yellow light and transmitted through the second phosphor layer. The further increasing of the distance makes phosphor layer move closer to LED chips, and thus more light is trapped and reflected inside the gap between the first phosphor layer and LED chips. This causes the junction temperature rise of phosphor layers and LED chips which may produce the low conversion efficiency. For the triple-layer structure, the process of the light propagation inside LEDs has similar tendency. Luminous flux also increases with the distance range within $0-0.6 \mathrm{~mm}$ and reduces at the position of the distance range within $0.6-$ $0.7 \mathrm{~mm}$. The lumen of triple layer structure reaches the highest value at the distance of $0.6 \mathrm{~mm}$. The simulation results show that the case of the dual-layer package has a better enhancement than the case of the triple-layer package at the beginning (see Fig. 2). In other words, the dual-layer configuration produces a higher percentage of extracted photons to the total light energy than triplelayer configuration.

\section{Conclusions}

In this research, the influence of the distance between phosphor layers as well as the number phosphor layers on the optical characteristics of remote phosphor package at the same CCT is analyzed and demonstrated in detail. The researched results revealed that the appropriate position of phosphor layer in remote phosphor package significantly improves the luminous flux and color rendering index of LEDs. The luminous flux remarkably enhances and achieves the maximum value at the distance of $0.6 \mathrm{~mm}$ for the case of trial-layer package and $0.1 \mathrm{~mm}$ for dual-layer package. Meanwhile, with the distance ranging from $0.1 \mathrm{~mm}$ to $0.7 \mathrm{~mm}$ of dual-layer package, the lumen output and the color quality of LEDs have slightly downward tendency. The reason of this issue is due to the increase of the trapping, the absorption, the re-scattering of light in LED package and the chemical change of the heated phosphor layer. Therefore, finding a suitable distance between phosphor layers in remote phosphor package is a key factor in designing high efficiency pc-LEDs.

This research is funded by Department of Science and Technology of Binh Thuan province under grant number DA02-01-2018.

\section{References}

1. Y. H. Won, H. S. Jang, K. W. Cho, Y. S. Song, D. Y. Jeon and H. K. Kwon, Opt. Lett. 34, 1 (2009).

2. K. J. Chen, C. C. Lin, H. V. Han, C. Y. Lee, S. H. Chien, K. Y. Wang, S. H Chiu, Z. Y. Tu, J. R. Li, T.
M Chen, X. Li, M. H. Shi and H. C. Kuo, IEEE J. Sel. Top. Quantum Electron 21, 1 (2015).

3. M. Zhang, X. H. Li, Z. X. Wang, Q. Y. Hu, H. J. Guo, Trans. Nonferrous Met. Soc. China 20, 115 (2010).

4. K.-C. Lee, S.-M. Kim and J.-H. Moon. Proc. SPIE 7784, Tenth International Conference on Solid State Lighting, 7784101 (2010).

5. L. Yang, Z.-C. Lv, Y. Jiaojiao and S. Liu, Appl. Opt. 52, 553 (2013).

6. C. Shen, J. Chu, F. Qian, X. Zou, C. Zhong, K. Li and S. Jin, J. Mod. Opt 59, 1199 (2012).

7. S. Chawla, T. Roy, K. Majumder and A. Yadav, J. Exp. Nanosci. 9776 (2014).

8. M. S. Jang, Y. H. Choi, S. Wu, T. G. Lim and J. S. Yoo, J. Inf. Display 17, 117 (2016).

9. Y. H. Won, H. S. Jang, K. W. Cho, Y. S. Song, D. Y. Jeon and H. K. Kwon, Opt. Lett. 34, 1 (2009).

10. Z. M. Chen, S. X. Jiang, R. H. Guo, B. J. Xin and D. G. Miao, Mater. Techno. 29, 198 (2014).

11. Z. M. Chen, S. X. Jiang, R. H. Guo, B. J. Xin and D. G. Miao, Mater. Techno. 31, 7 (2016).

12. M. Zhang, X. -H. Li, Z. -X. Wang, Q. -Y. Hu, H. -J Guo, Trans. Nonferrous Met. Soc. China, 20, 115 (2010).

13. Z.-Y Liu, S. Liu, K. Wang, and X.-B Luo, Appl. Opt. 49, 247 (2010).

14. K.-C. Lee, S.-M. Kim and J.-H. Moon, In Proceedings of Tenth International Conference on Solid State Lighting, San Diego, USA, 7784 (2010).

15. N. D. Q. Anh, M. F. Lai, H. Y. Ma, H. Y. Lee, J. Chin. Inst. Eng. 38, 297 (2015).

16. M. F. Lai, N. D. Q. Anh, H. Y. Ma, H. Y. Lee, J. Chin. Inst. Eng., 39, 1 (2016).

17. K. J. Chen, H. C. Chen, M. H. Shih, C.H. Wang, H. H. Tsai, S. H. Chien, C. C. Lin, H. C. Kuo, J. Light. Technol, 31, 1941 (2013).

18. H. C. Chen, K. J. Chen, C. C. Lin, C. H. Wang, C. C. Yeh. H. H. Tsai, M. H. Shih, and H. C. Kuo, Microelectron. Reliab. 52, (2012).

19. S. Yu, Z. Li, G. Liang, Y. Tang, B. Yu, K. Chen, Photon. Res. 4, 140 (2016).

20. F. W. Mont, J. K. Kim, M. F. Schubert, H. Luo, E. F. Schubert, and R. W. Siegel, J. Appl. Phys., 103, 083120 (2007).

21. N. D. Q. Anh, H. Y. Lee, T. T. Phuong, N. H. K. Nhan, T. H. Q. Minh and T. H. Ly, J. Chin. Inst. Eng, 40, 1 (2017).

22. N. D. Q. Anh and H. Y. Lee, J. Chin. Inst. Eng 39, 871 (2016).

23. S. Liu and X. B. Luo, LED Packaging for Lighting Applications: Design, Manufacturing and Testing, John Wiley \& Sons, 2011, pp. 67-144 